Pacific Journal of Mathematics

THE TWO-DIMENSIONAL DIOPHANTINE APPROXIMATION 


\title{
THE TWO-DIMENSIONAL DIOPHANTINE APPROXIMATION CONSTANT. II
}

\author{
T. W. CUSICK
}

\begin{abstract}
Given real numbers $\alpha$ and $\beta$, let $c_{1}(\alpha, \beta)$ denote the Diophantine approximation constant for the linear form $x+\alpha y+\beta z$ and let $c_{2}(\alpha, \beta)$ denote the corresponding dual constant for the simultaneous approximation of $\alpha$ and $\beta$. The paper gives various results about these constants in the case where $\alpha$ and $\beta$ lie in some real cubic field. For example, it is shown that the suprema of $c_{1}(\alpha, \beta)$ and $c_{2}(\alpha, \beta)$, taken over all $\alpha, \beta$ such that $1, \alpha, \beta$ is an integral basis for a real cubic field, are equal, and a necessary and sufficient condition for this common value to be equal to $2 / 7$ is given.
\end{abstract}

1. Introduction. There is associated with each real number $\alpha$ a constant $c(\alpha)$ defined to be the infimum of those $c>0$ such that the inequality

$$
|x(\alpha x-y)|<c
$$

has infinitely many solutions in integers $x, y$ with $x \neq 0$. A well known theorem of Hurwitz states that $\sup c(\alpha)$, where the supremum is taken over all real numbers $\alpha$, is equal to $1 / \sqrt{5}$, and that $c(\alpha)=1 / \sqrt{5}$ only for certain numbers, such as $\frac{1}{2}(1+\sqrt{5})$, in the algebraic extension $Q(\sqrt{5})$ of the rational field $Q$.

In the theory of simultaneous Diophantine approximation, there are two well known constants associated with each pair of real numbers $\alpha, \beta$. One constant, which I denote by $c_{1}(\alpha, \beta)$, is defined to be the infimum of those $c>0$ such that the inequality

$$
|x+\alpha y+\beta z| \max \left(y^{2}, z^{2}\right)<c
$$

has infinitely many solutions in integers $x, y, z$ with $y$ and $z$ not both zero. The other constant, which I denote by $c_{2}(\alpha, \beta)$, is defined to be the infimum of those $c>0$ such that the inequality

$$
\max \left(|x|(\alpha x-y)^{2},|x|(\beta x-z)^{2}\right)<c
$$

has infinitely many solutions in integers $x, y, z$ with $x \neq 0$.

Define $L=\sup c_{1}(\alpha, \beta)$ and $S=\sup c_{2}(\alpha, \beta)$, where the suprema are taken over all pairs of real numbers $\alpha, \beta$. It is a well known unsolved problem to evaluate $S$. Cassels [4] showed that $S \geq 2 / 7$. Davenport [11] proved that $L=S$ and [10] that $S<.384$.

Define $L^{*}=\sup c_{1}(\alpha, \beta)$ and $S^{*}=\sup c_{2}(\alpha, \beta)$, where the suprema are taken over all $\alpha, \beta$ such that $1, \alpha, \beta$ is a basis of a real cubic field. It is 
often the case that for such $\alpha, \beta$ we have $c_{1}(\alpha, \beta)=c_{2}(\alpha, \beta)$ (see [7], especially p. 187, and [8]), so it is natural to conjecture that $L^{*}=S^{*}$. This is indeed the case, and in fact $L^{*}=S^{*}=2 / 7$ holds. The work of Cassels [4] gives the inequality $S^{*} \geq 2 / 7$, and the opposite inequality follows from a difficult theorem of Woods [15] - the argument is given by Adams [2]. The inequalities $S^{*} \geq 2 / 7$ and $L^{*} \geq 2 / 7$ can also be proved by using an idea due to Davenport [11]; this is carried out in $\$ 4$ below. Finally, the inequality $L^{*} \leq 2 / 7$ is proved in $\S 4$; the key ingredient is again the result of Woods [15].

The above results suggest the main unsolved problem in this subject:

Conjecture. $L=S=2 / 7$.

This paper does not deal with the conjecture, but instead gives more results for the case in which only numbers in a cubic field are considered. Define $L^{\prime}=\sup c_{1}(\alpha, \beta)$ and $S^{\prime}=\sup c_{2}(\alpha, \beta)$, where the suprema are taken over all $\alpha, \beta$ such that $1, \alpha, \beta$ is an integral basis of a real cubic field. A main result of this paper is the following:

THEOREM 1. We have $L^{\prime}=S^{\prime} \leq 2 / 7$; the inequality is actually an equality if and only if Hypothesis A below is true.

The Hypothesis A mentioned in Theorem 1 was first stated in my paper [9], p. 298. In that paper it was proved that if $\theta=2 \cos (2 \pi / 7)$ denotes one of the real roots of $x^{3}+x^{2}-2 x-1=0$ (so $Q(\theta)$ is the cubic field of discriminant 49 , which is the smallest possible positive discriminant) and if $1, \alpha, \beta$ is any integral basis of $Q(\theta)$, then $c_{1}(\alpha, \beta) \leq$ $2 / 7$; furthermore, $\sup c_{1}(\alpha, \beta)=2 / 7$ for such integral bases $1, \alpha, \beta$ if and only if the following hypothesis is true:

Hypothesis A. The number $\theta=2 \cos (2 \pi / 7)$ has a continued fraction expansion $\left[a_{1}, a_{2}, \ldots\right]$ such that given any integer $N$, either a partial quotient pattern

$$
\left(a_{n}, a_{n+1}, a_{n+2}, a_{n+3}\right)=\left(N_{1}, 1,1, N_{2}\right),
$$

where $N_{1}>N$ and $N_{2}>N$, occurs for some $n$; or a partial quotient pattern

$$
\left(a_{n}, a_{n+1}, a_{n+2}\right)=\left(N_{1}, 2, N_{2}\right),
$$

where $N_{1}>N$ and $N_{2}>N$, occurs for some $n$. 
We have very little knowledge about the continued fraction expansions of algebraic numbers of degree greater than 2 , so there is at present no hope of deciding the truth of Hypothesis A. However, Hypothesis A is plausible if one agrees with the widely held but intractable conjecture that the continued fraction for any algebraic number of degree greater than 2 resembles, in a statistical sense, the continued fractions for almost all real numbers (so that in particular any given finite sequence of integers will occur in the sequence of partial quotients). Furthermore, independently of the validity of Hypothesis $\mathrm{A}$, it is possible to compute lower bounds for $L^{\prime}$ and $S^{\prime}$ which are extremely close to $2 / 7$ (see $\$ 6$ below).

2. Upper bounds for $c_{1}(\alpha, \beta)$. Throughout this paper, if $\delta$ is an element of a real cubic field, then $\delta, \delta^{\prime}, \delta^{\prime \prime}$ are the conjugates of $\delta$. The norm $\delta \delta^{\prime} \delta^{\prime \prime}$ of $\delta$ is denoted by $N(\delta)$.

We need only consider totally real cubic fields in proving Theorem 1, because the nontotally real case (which is much simpler) is taken care of in $\S 5$ below. Let $\alpha_{0}, \alpha_{1}, \alpha_{2}$ be a basis for a totally real cubic field and let

$$
M=\left\{\xi=\alpha_{0} x+\alpha_{1} y+\alpha_{2} z: x, y, z \text { integers }\right\}
$$

be the module with basis $\alpha_{0}, \alpha_{1}, \alpha_{2}$. We define

$$
m_{+}(M)=\inf _{\substack{\xi \operatorname{in} M, \xi>0 \\ N(\xi)>0}} N(\xi) \text { and } m_{-}(M)=\inf _{\substack{\xi \operatorname{in} M, \xi>0 \\ N(\xi)<0}}|N(\xi)|
$$

and define $m_{+}\left(M^{*}\right)$ and $m_{-}\left(M^{*}\right)$ analogously for the dual module $M^{*}$ of $M$. It can happen that $m_{+}(M) \neq m_{-}(M)$; of course, there is no corresponding possibility for modules over a nontotally real cubic field, which partly explains why that situation is so much simpler.

If $1, \alpha, \beta$ is a basis for a totally real cubic field, we define the binary quadratic form $f(x, y)$ by

$$
\text { (1) } f(x, y)=\left(\left(\beta^{\prime \prime}-\beta\right) x+\left(\alpha-\alpha^{\prime \prime}\right) y\right)\left(\left(\beta-\beta^{\prime}\right) x+\left(\alpha^{\prime}-\alpha\right) y\right) \text {. }
$$

It is easy to see that the discriminant of $f(x, y)$ is equal to the discriminant of the module $M$ with basis $1, \alpha, \beta$, so $f(x, y)$ is an indefinite form.

Our first lemma is a result from [8], which gives formulas for $c_{1}(\alpha, \beta)$ and $c_{2}(\alpha, \beta)$ in terms of certain quantities depending on the form (1). We need the following notation: Define the sets $U, U^{-1}, V, V^{-1}$ by

$$
\begin{aligned}
U & =\{x:|x| \leq 1 \text { and } f(x, 1) \geq 0\}, \\
U^{-1} & =\{y:|y| \leq 1 \text { and } f(1, y) \geq 0\}
\end{aligned}
$$


and

$$
\begin{aligned}
V & =\{x:|x| \leq 1 \text { and } f(x, 1) \leq 0\}, \\
V^{-1} & =\{y:|y| \leq 1 \text { and } f(1, y) \leq 0\} .
\end{aligned}
$$

Define the numbers $f_{U}$ and $f_{V}$ by

$$
f_{U}=\max \left(\max _{\substack{|x| \leq 1 \\ x \text { in } U}} f(x, 1), \max _{\substack{|y| \leq 1 \\ y \text { in } U^{-1}}} f(1, y)\right)
$$

and

$$
f_{V}=\max \left(\max _{\substack{|x| \leq 1 \\ x \text { in } V}}|f(x, 1)|, \max _{\substack{|y| \leq 1 \\ y \text { in } V^{-1}}}|f(1, y)|\right) .
$$

Lemma 1. Suppose $1, \alpha, \beta$ is a basis for a totally real cubic field. Let $M$ denote the module with basis $1, \alpha, \beta$ and let $D_{M}$ denote the discriminant of $M$. Let $M^{*}$ denote the dual module of $M$ and define the quadratic form $f(x, y)$ by (1). Then

$$
c_{1}(\alpha, \beta)=\min \left(\frac{m_{+}(M)}{f_{V}}, \frac{m_{-}(M)}{f_{U}}\right)
$$

and

$$
c_{2}(\alpha, \beta)=\min \left(\frac{D_{M} m_{+}\left(M^{*}\right)}{f_{U}}, \frac{D_{M} m_{-}\left(M^{*}\right)}{f_{V}}\right)
$$

Define

$$
\nu(f)=\max \left(\max _{|x| \leq 1}|f(x, 1)|, \max _{|y| \leq 1}|f(1, y)|\right) .
$$

If $m_{+}(M)=m_{-}(M)$, then

$$
c_{1}(\alpha, \beta)=m_{+}(M) / \nu(f) .
$$

If $m_{+}\left(M^{*}\right)=m_{-}\left(M^{*}\right)$, then

$$
c_{2}(\alpha, \beta)=D_{M^{2}} m_{+}\left(M^{*}\right) / \nu(f) .
$$

Proof. This is Theorem 2 of [8] with the special cases (5) and (6) written out; these last follow from the fact that $\nu(f)=\max \left(f_{U}, f_{V}\right)$.

We shall need the following simple lower bound for the quantity $\nu(f)$ defined in (4).

LemMa 2. Define $f(x, y)$ by (1) and let $\nu(f)$ be given by (4). Let $D$ denote the discriminant of the module with basis $1, \alpha, \beta$; then $\nu(f) \geq \frac{1}{2} D^{1 / 2}$. 
Proof. A special case of this result is proved in [9], Lemma 2. The general proof uses the same method, but we give it here for completeness.

We label the coefficients of $f(x, y)$ so that

$$
f(x, y)=a x^{2}+b x y+c y^{2} .
$$

As was already remarked, an easy calculation gives

$$
b^{2}-4 a c=D>0 \text {. }
$$

The form $f(x, y)$ is indefinite, so the only possible values for which the maximum of $f(x, 1)$ in $|x| \leq 1$ could occur are \pm 1 or $-b / 2 a$, and the only possible values of $y$ for which the maximum of $|f(1, y)|$ in $|y| \leq 1$ could occur are \pm 1 and $-b / 2 c$. by (4)

We may assume $a c<0$, for if $a c>0$ then (8) implies $|b|>D^{1 / 2}$, so

$$
\nu(f) \geq \max (|a+b+c|,|a-b+c|) \geq|b|>D^{1 / 2},
$$

which is stronger than the inequality in the lemma.

We consider several cases, supposing first that $|b| \leq|2 a|$ and $|b| \leq$ $|2 c|$. Then (4) implies

$$
\begin{aligned}
\nu(f) & \geq \max (|f(-b / 2 a, 1)|,|f(1,-b / 2 c)|) \\
& =\max (D /|4 a|, D /|4 c|) .
\end{aligned}
$$

Now (8) and the fact that $a c<0$ imply $|4 a c| \leq D$, so either $|a| \leq \frac{1}{2} D^{1 / 2}$ or $|c| \leq \frac{1}{2} D^{1 / 2}$; combining this with (9) gives the lemma.

Next suppose $|b|>|2 a|$ and $|b| \leq|2 c|$. Here (4) implies

$$
\nu(f) \geq \max (|a+b+c|,|a-b+c|, D /|4 c|) .
$$

If $|c| \leq \frac{1}{2} D^{1 / 2}$, then (10) immediately gives the lemma, so we may assume $|c|>\frac{1}{2} D^{1 / 2}$. Now $|2 c| \geq|b|>|2 a|$ by hypothesis, so with the appropriate choice of the \pm sign we have

$$
|a \pm b+c|=|b|+|c|-|a|>|a|+|c|>\frac{1}{2} D^{1 / 2}
$$

and the lemma follows from (10).

An argument exactly analogous to the above works if $|b| \leq|2 a|$ and $|b|>|2 c|$, so the only remaining case is that in which $|b|>|2 a|$ and $|b|>|2 c|$. Since $b^{2}+4|a c|=D$ by (8), in this case we have $b^{2}>D / 2$. Thus with an appropriate choice of the \pm sign we have

$$
\nu(f) \geq|a \pm b+c| \geq|b|>D^{1 / 2} / \sqrt{2},
$$

which is stronger than the inequality in the lemma. 
We can now prove some upper bounds for the constants $c_{1}(\alpha, \beta)$ in the case where $1, \alpha, \beta$ is an integral basis.

THEOREM 2. Let $1, \alpha, \beta$ be an integral basis for a totally real cubic field and let $M$ denote the module with basis $1, \alpha, \beta$ and discriminant $D$; then

$$
c_{1}(\alpha, \beta) \leq 2 m_{-}(M) / D^{1 / 2}
$$

and

$$
c_{1}(\alpha, \beta) \leq 2 / D^{1 / 4} .
$$

If $m_{-}(M) \leq \frac{1}{4} D^{1 / 2}$, then

$$
c_{1}(\alpha, \beta) \leq 1 / D^{1 / 4} .
$$

Proof. We define the quadratic form $f(x, y)$ by (1), and label its coefficients so that (7) holds. Then (8) is true, as before.

The proof will resemble that of Lemma 2, but with some complications. We dispose of the easiest inequality, given by (11), first. Since $1, \alpha$, $\beta$ is an integral basis, we have $m_{+}(M)=1$, so (2) gives

$$
c_{1}(\alpha, \beta)=\min \left(1 / f_{V}, m_{-}(M) / f_{U}\right) .
$$

Since the quantity $\nu(f)$ defined by (4) satisfies $\nu(f)=\max \left(f_{U}, f_{V}\right)$, it follows from (14) and Lemma 2 that

$$
c_{1}(\alpha, \beta) \leq m_{-}(M) / \nu(f) \leq 2 m_{-}(M) / D^{1 / 2},
$$

which proves (11).

To prove (12), we shall need the estimate

$$
m_{-}(M) \leq D^{1 / 2},
$$

which is a result of Chalk [6] (or see Cassels [5, p. 330]).

We divide the proof of (12) into three cases; in the first, we assume that $|b| \leq|2 a|$ and $|b| \leq|2 c|$. By (8), this implies $a c<0$. We suppose that $a>0$ and $c<0$; an exactly analogous argument works if $a<0$ and $c>0$.

Under our assumptions we have

$$
f_{U} \geq f(1,-b / 2 c)=D /|4 c| \text { and } f_{V} \geq|f(-b / 2 a, 1)|=D / 4 a \text {. }
$$

It follows from (14) that (12) holds if $|a|^{-1} \geq 2 / D^{3 / 4}$, and from (14) and (15) that (12) holds if $|c|^{-1} \geq 2 / D^{1 / 4}$. At least one of these inequalities is true, for if both were false we would have $|a c|^{-1}<4 / D$, which contradicts $b^{2}-4 a c=D \geq|4 a c|$. 
For our second case, we assume that $|b|>|2 a|$ and $|b| \leq|2 c|$. Parallel arguments work if $|b| \leq|2 a|$ and $|b|>|2 c|$. First suppose that $a c>0$, say $a>0$ and $c>0$ (a simpler method works if $a$ and $c$ are negative). If $b>0$, then $a+b+c>0$ so $x=1$ is in $U$ and therefore

$$
f_{U} \geq|a|+|b|+|c| \text {. }
$$

If, on the other hand, $b<0$, then $a-b+c>0$ so $x=-1$ is in $U$ and again (16) holds. Whatever the sign of $b, c>0$ implies

$$
f_{V} \geq|f(1,-b / 2 c)|=D / 4 c \text {. }
$$

If $D / 4 c \geq \frac{1}{2} D^{1 / 4}$, then $1 / f_{V} \leq 2 / D^{1 / 4}$ and (14) gives (12). So we may assume $c>\frac{1}{2} D^{3 / 4}$. Then (16) implies $f_{U}>\frac{1}{2} D^{3 / 4}$, so $m_{-}(M) / f_{U}<$ $2 D^{1 / 2} / D^{3 / 4}$ by (15), and again (14) gives (12).

Next suppose that $|b|>|2 a|,|b| \leq|2 c|$ and $a c<0$. We consider the situation with $a>0$ and $c<0$ first. If $b>0$, then $|2 c| \geq|b|>|2 a|$ implies $a-b+c<0$, so $x=-1$ is in $V$ and

$$
f_{V} \geq|b|+|c|-|a| \text {. }
$$

If, on the other hand, $b<0$, then $a+b+c<0$ so $x=1$ is in $V$ and again (17) holds. Whatever the sign of $b, c<0$ implies $f_{U} \geq D /|4 c|$. If $D /|4 c| \geq \frac{1}{2} D^{3 / 4}$, then $m_{-} / f_{U} \leq 2 / D^{1 / 4}$ by (15), and (14) gives (12). So we may assume $|c|>\frac{1}{2} D^{1 / 4}$. Now since $|b|>|a|$, (17) implies $f_{V}>|c|$, so $1 / f_{V}<2 / D^{1 / 4}$ and again (14) gives (12). The situation with $a<0$ and $c>0$ is taken care of similarly, but we use $f_{U} \geq|b|+|c|-|a|$ in place of (17).

For our third case, we assume that $|b|>|2 a|$ and $|b|>|2 c|$. First suppose that $a c>0$, so $a$ and $c$ have the same sign. We have $|b|>|a|+$ $|c|$, so $f(1,1)=a+b+c$ and $f(-1,1)=a-b+c$ have opposite signs. If $b<0$, this means -1 is in $U$ and 1 is in $V$; if $b>0$, then -1 is in $V$ and 1 is in $U$. In either case, we have

$$
f_{U} \geq|a|+|b|+|c| \text { and } f_{V} \geq-|a|+|b|-|c|,
$$

so by (14) and (15) we have (12) if either of the inequalities

$$
|a|+|b|+|c|<\frac{1}{2} D^{3 / 4} \text { and }-|a|+|b|-|c|<\frac{1}{2} D^{1 / 4}
$$

is false. Thus we may assume that both of the inequalities in (18) are true, and multiplying them together gives

$$
|b|^{2}-(|a|+|c|)^{2}<\frac{1}{4} D
$$


We have $D=b^{2}-4 a c=b^{2}-4|a c|$, so the above inequality becomes

$$
\frac{3}{4} D<(|a|-|c|)^{2} \text { or } \frac{\sqrt{3}}{2} D^{1 / 2}<|| a|-| c|| \text {. }
$$

But now the second inequality in (18) and our assumption $|b|>$ $2 \max (|a|,|c|)$ give

$$
\frac{1}{2} D^{1 / 4}>|b|-|a|-|c| \leq|| a|-| c||>\frac{\sqrt{3}}{2} D^{1 / 2},
$$

which is a contradiction.

Finally, we suppose that $|b|>|2 a|,|b|>|2 c|$ and $a c<0$. As before, we have $|b|>|a|+|c|$, so $a+b+c$ and $a-b+c$ have opposite signs. We suppose $a>0$ and $c<0$ (the same reasoning works if $a<0$ and $c>0$ ). If $b<0$, we have -1 in $U$ and 1 in $V$; if $b>0$, we have -1 in $V$ and 1 in $U$. In either case, we obtain

$$
f_{U} \geq|a|+|b|-|c| \text { and } f_{V} \geq-|a|+|b|+|c|,
$$

so by (14) we have (12) if

$$
-|a|+|b|+|c|<\frac{1}{2} D^{1 / 4}
$$

is false. Thus we can assume that (19) is true, and hence (since $|b|>|2 a|$ ) we have $\max (|a|,|c|)<\frac{1}{2} D^{1 / 4}$. Therefore $4|a c|<D^{1 / 2}$, so that (8) implies $|b|>\left(D-D^{1 / 2}\right)^{1 / 2}$. Combining this last inequality with (19) gives

$$
\left(D-D^{1 / 2}\right)^{1 / 2}-\frac{1}{2} D^{1 / 4}<-|a|+|b| \leq-|a|+|b|+|c|<\frac{1}{2} D^{1 / 4}
$$

which is a contradiction. This completes the proof of (12).

To prove (13), we follow the above proof of (12), but use the assumption $m_{-} \leq \frac{1}{4} D^{1 / 2}$ in place of (15) throughout the proof. This completes the proof of Theorem 2 .

3. Proof of Theorem 1. If $D \geq 2429$ and $1, \alpha, \beta$ is an integral basis for a totally real cubic field $F$ of discriminant $D$, then by (12) in Theorem 2 we have

$$
c_{1}(\alpha, \beta) \leq 2(2429)^{-1 / 4}=.284887<2 / 7 .
$$

Let $M$ be the algebraic integers of $F$; by (13) in Theorem 2, we have $c_{1}(\alpha, \beta) \leq D^{-1 / 4}$ if

$$
m_{-}(M)<\frac{1}{4} D^{1 / 2}
$$


holds. Calculation shows that $(21)$ is true for all $D<2429$. (The table of Angell [3] was used. For $D<2429$, each discriminant corresponds to a unique cubic field (apart from conjugate fields). There are 75 such fields, but $m_{+}(M)=m_{-}(M)=1$ for all but 33 of the fields. Inequality (21) holds comfortably for all 33.) Therefore

$$
c_{1}(\alpha, \beta) \leq(169)^{-1 / 4}=.277350
$$

for all $D$ such that $2429>D \geq 169$. Hence (20) holds for all $D$ except perhaps $D=49,81$ or 148 . Since $m_{-}(M)=1$ for all three of these fields, it follows from (11) in Theorem 2 that (20) holds except possibly when $D=49$. We know (see $\S 6$ below) that we can choose $\alpha, \beta$ in the field with discriminant 49 (this is the field $Q(\theta)$ mentioned in the Introduction) so that $(20)$ is false. Hence we have shown that in calculating $L^{\prime}$, we need only consider the field $Q(\theta)$. Hence it follows from the theorem of [9] that

$$
L^{\prime} \leq 2 / 7 \text {, }
$$

with equality if and only if Hypothesis $\mathrm{A}$ in the Introduction is true.

In order to complete the proof of Theorem 1, it suffices to show that $L^{\prime}=S^{\prime}$; to do this we need an inequality like (20) for the constants $c_{2}(\alpha, \beta)$.

Let $M^{*}$ denote the dual module of $M$. Given any module $M$, let $D_{M}$ denote its discriminant. We have $D_{M^{*}}=D_{M}^{-1}=D^{-1}$, so inequality (14) gives

$$
m_{-}\left(M^{*}\right) \leq D^{-1 / 2} \text {. }
$$

We also have $m_{+}\left(M^{*}\right)=D^{-1}$ since $M$ is the module of all algebraic integers in $F$. Therefore (3) in Lemma 1 gives

$$
c_{2}(\alpha, \beta)=\min \left(1 / f_{U}, D m_{-}\left(M^{*}\right) / f_{V}\right) .
$$

By imitating the proof of Theorem 2 and using (22) and (23) in place of (15) and (14), respectively, we find that

$$
c_{2}(\alpha, \beta) \leq 2 m_{-}\left(M^{*}\right) D^{1 / 2}
$$

and

$$
c_{2}(\alpha, \beta) \leq 2 / D^{1 / 4} .
$$

We also find that if $m_{-}\left(M^{*}\right) \leq\left(4 D^{1 / 2}\right)^{-1}$, then

$$
c_{2}(\alpha, \beta) \leq 1 / D^{1 / 4} \text {. }
$$

These last three inequalities are the analogues of (11), (12) and (13), respectively. 
Using (25), we see that inequality (20) also holds for $c_{2}(\alpha, \beta)$ when $D \geq 2429$. Using (26) and our argument above, we find that (20) holds for $c_{2}(\alpha, \beta)$ except perhaps when $D$ is 49,81 or 169 ; for this we need to verify that $m_{-}\left(M^{*}\right) \leq\left(4 D^{1 / 2}\right)^{-1}$ for all $D<2429$, but this is a straightforward calculation.

Finally, by using (24) and the fact that $m_{+}\left(M^{*}\right)=m_{-}\left(M^{*}\right)=D^{-1}$ for $D=49,81$ or 169 , we prove that in calculating $S^{\prime}$ we need only consider the field $Q(\theta)$. Since $c_{1}(\alpha, \beta)=c_{2}(\alpha, \beta)$ always holds in $Q(\theta)$ (by (5) and (6) in Lemma 1), we have $S^{\prime}=L^{\prime}$.

4. Proof that $L^{*}=2 / 7$. Let $I$ denote the algebraic integers in $Q(\theta), \theta=2 \cos (2 \pi / 7)$ (this is the totally real cubic field of discriminant 49 mentioned in the Introduction). Thus $D_{I}=49$.

Let $N$ be any large positive integer. According to a theorem of Davenport [11, §2], we can find a basis $\mu_{0}, \mu_{1}, \mu_{2}$ of $I$ such that for any $\varepsilon>0$

$$
\mu_{0}^{\prime} \approx \mu_{0}^{\prime \prime}=O\left(N^{\varepsilon}\right)
$$

and

$$
\mu_{1}^{\prime} \approx \mu_{2}^{\prime} \approx \mu_{1}^{\prime \prime} \approx-\mu_{2}^{\prime \prime}=N+O\left(N^{\varepsilon}\right) .
$$

Here we are actually using a slightly generalized form of Davenport's idea, as given in Cassels [5, p. 16].

Now we define $\alpha, \beta$ and $\delta>0$ (we change the signs of $\mu_{0}, \mu_{1}, \mu_{2}$ if necessary to obtain $\delta>0$ ) uniquely by the equations

$$
\begin{aligned}
\mu_{0}+\mu_{1} \alpha+\mu_{2} \beta & =\delta, \\
\mu_{0}^{\prime}+\mu_{1}^{\prime} \alpha+\mu_{2}^{\prime} \beta & =0, \\
\mu_{0}^{\prime \prime}+\mu_{1}^{\prime \prime} \alpha+\mu_{2}^{\prime \prime} \beta & =0 .
\end{aligned}
$$

We obtain

$$
\delta=D_{I}^{1 / 2}\left(\mu_{1}^{\prime} \mu_{2}^{\prime \prime}-\mu_{1}^{\prime \prime} \mu_{2}^{\prime}\right)^{-1} \approx(7 / 2) N^{-2}
$$

by (27). It follows that $\delta$ is in $Q(\theta)$, and $\alpha$ and $\beta$ are also. We define the module $M$ by

$$
M=\{x+\alpha y+\beta z: x, y, z \text { integers }\} .
$$

It follows from equations (28) that $\delta^{-1}, \alpha \delta^{-1}, \beta \delta^{-1}$ is the dual basis of $\mu_{0}$, $\mu_{1}, \mu_{2}$; hence

$$
M=\delta I^{*}
$$


and

$$
D_{M}=\delta^{6} / D_{I}=\delta^{6} / 49 \text {. }
$$

It is easy to see that the dual module $I^{*}=\left(\left(\theta-\theta^{\prime}\right)\left(\theta-\theta^{\prime \prime}\right)\right)^{-1} I$. The norm of $\left(\theta-\theta^{\prime}\right)\left(\theta-\theta^{\prime \prime}\right)$ is -49 , so we have from (30)

$$
m_{+}(M)=m_{+}\left(\delta I^{*}\right)=\delta^{3} / 49=m_{-}(M) .
$$

It follows from (28) that

$$
\mu_{1}=\delta\left(\beta^{\prime}-\beta^{\prime \prime}\right) D_{M}^{-1 / 2} \text { and } \mu_{2}=\delta\left(\alpha^{\prime \prime}-\alpha^{\prime}\right) D_{M}^{-1 / 2} .
$$

Therefore if $f(x, y)$ is defined by (1), we have from (27) and (31)

$$
\begin{aligned}
f(x, y) & =D_{M} \delta^{-2}\left(\mu_{1}^{\prime} x+\mu_{2}^{\prime} y\right)\left(\mu_{1}^{\prime \prime} x+\mu_{2}^{\prime \prime} y\right) \\
& \approx D_{M} \delta^{-2} N^{2}\left(x^{2}-y^{2}\right)=\left(\delta^{4} N^{2} / 49\right)\left(x^{2}-y^{2}\right) .
\end{aligned}
$$

If we apply (29), (32) and (33) to formula (5) of Lemma 1, we obtain

$$
c_{1}(\alpha, \beta) \approx\left(\delta^{3} / 49\right) /\left(\delta^{4} N^{4} / 49\right) \approx 2 / 7
$$

as $N \rightarrow \infty$. Hence we have $L^{*} \geq 2 / 7$. A similar argument using (6) instead of (5) gives an alternative proof that $S^{*} \geq 2 / 7$ (see the Introduction). Adams [1] gave a similar proof that $S^{*} \geq 2 / 7$, also using Davenport's idea.

Now we shall prove $L^{*} \leq 2 / 7$. For this we need two more lemmas.

LEMMA 3. Let $f(x, y)=a x^{2}+b x y+c y^{2}$ be a quadratic form with discriminant $D=b^{2}-4 a c>0$. Let $B_{r}$ be the open square $\max (|x|,|y|)<r$, and let $\sigma, \tau$ be any positive numbers. Then provided $r^{2}>\sigma \tau^{1 / 2}$, at least one of the curves

$$
f(x, y)=\frac{1}{2} \sigma D^{1 / 2}, \quad f(x, y)=-\frac{1}{2} \sigma \tau D^{1 / 2}
$$

intersects $B_{r}$.

Proof. This is Proposition 2 of Adams' paper [1].

Now let $f_{U}$ and $f_{V}$ denote the numbers defined in $\S 2$ above.

LEMMa 4. Let $f(x, y)$ be any indefinite binary quadratic form. Then

$$
\inf \left\{r^{2}: f(x, y)= \pm 1 \text { intersects } B_{r}\right\}=f_{U}^{-1}
$$

and

$$
\inf \left\{r^{2}: f(x, y)=-1 \text { intersects } B_{r}\right\}=f_{V}^{-1}
$$


Proof. This is Lemma 6 of my paper [8].

Let $M$ denote any module $\{x+\alpha y+\beta z\}$, where $1, \alpha, \beta$ is a basis of a totally real cubic field. Define the quadratic form $f(x, y)$ by (1), so the discriminant of $f(x, y)$ is $D_{M}$. Take

$$
\sigma=2 m_{-}(M), \quad \tau=m_{+}(M) / m_{-}(M)
$$

so $\sigma \tau^{1 / 2}=2\left(m_{+}(M) m_{-}(M)\right)^{1 / 2}=g(M)$, say. Let $r>0$ be any number such that $r^{2}>g(M)$. By Lemma 3 we have either

$$
m_{-}(M) D_{M}^{1 / 2} \inf \left\{r^{2}: f(x, y)=+1 \text { intersects } B_{r}\right\} \leq g(M)
$$

or

$$
m_{+}(M) D_{M}^{1 / 2} \inf \left\{r^{2}: f(x, y)=-1 \text { intersects } B_{r}\right\} \leq g(M) .
$$

In either case, it follows from Lemma 4 and (2) in Lemma 1 that $c_{1}(\alpha, \beta) \leq g(M) / D_{M}^{1 / 2}$ and hence

$$
L^{*} \leq \sup 2\left(m_{+}(M) m_{-}(M) / D_{M}\right)^{1 / 2},
$$

where the supremum is taken over all modules $M$ and all totally real cubic fields; actually, equality holds in (34) but we do not need this.

It follows from a theorem of Woods [15] that $m_{+}(M) m_{-}(M) \leq$ $D_{M} / 49$ always holds, so (34) gives $L^{*} \leq 2 / 7$. This completes the proof of:

THEOREM 3. $L^{*}=2 / 7$.

5. The case of nontotally real cubic fields. Define $L_{N}^{*}=\sup c_{1}(\alpha, \beta)$ and $S_{N}^{*}=\sup c_{2}(\alpha, \beta)$, where the suprema are taken over all $\alpha, \beta$ such that $1, \alpha, \beta$ is a basis of a nontotally real cubic field. Define $L_{N}^{\prime}$ and $S_{N}^{\prime}$ similarly, except that the suprema are taken over all $\alpha, \beta$ such that $1, \alpha, \beta$ is an integral basis of a nontotally real cubic field.

The nontotally real case is much simpler than the totally real case. In fact, it is not difficult to prove:

THEOREM 4. We have

$$
L_{N}^{*}=S_{N}^{*}=(23)^{-1 / 2}=.2085 \ldots
$$

and

$$
L_{N}^{\prime}=S_{N}^{\prime}=c_{1}\left(\gamma^{2}, \gamma^{2}-\gamma\right)=.1711 \ldots,
$$

where $\gamma$ is the real root of $x^{3}-x-1=0$. 
The inequalities $L_{N}^{*} \geq(23)^{-1 / 2}$ and $S_{N}^{*} \geq(23)^{-1 / 2}$ go back to Davenport and Mahler [12]. The inequality $S_{N}^{*} \leq(23)^{-1 / 2}$ was proved by Adams [1]. The inequality $L_{N}^{*} \leq(23)^{-1 / 2}$ can be proved in a similar way using [7, Theorem 1] or [8, Theorem 1].

The evaluation of $L_{N}^{\prime}$ and $S_{N}^{\prime}$ is easily accomplished by using the method of [9]. The binary quadratic forms considered in that paper were indefinite, but the corresponding forms here are definite. This simplifies the application of the method of [9]. The situation is further simplified by the fact that $c_{1}(\alpha, \beta)=c_{2}(\alpha, \beta)$ whenever $1, \alpha, \beta$ is an integral basis of the field $Q(\gamma)$ with discriminant -23 . This follows from [7, Corollary 1, p. 187] because $1, \gamma, \gamma^{2}$ is an integral basis for $Q(\gamma)$.

6. Lower bounds for $L^{\prime}$ and $S^{\prime}$. Since $L^{\prime}=S^{\prime}$ by Theorem 1 , we need only consider $L^{\prime}$. Theorem 1 also says that $L^{\prime}=2 / 7$ if and only if Hypothesis $A$ in the Introduction is true. Let $\theta=2 \cos (2 \pi / 7)$ have the continued fraction expansion $\left[a_{1}, a_{2}, \ldots\right]$. Hypothesis $\mathrm{A}$ is concerned with the occurrence of patterns

$$
N_{1}, 1,1, N_{2} \text { or } N_{1}, 2, N_{2} \quad\left(N_{1}>N \text { and } N_{2}>N\right)
$$

of successive $a_{i}$ 's in the sequence of partial quotients $a_{l}$, where $N$ is a large integer.

We cannot decide whether such patterns (35) occur with $N$ arbitrarily large. However, it is clear from the work in [9] that if we can exhibit any patterns of form (35) with $N$ large, then we can find $\alpha, \beta$ such that $c_{1}(\alpha, \beta)$ is near $2 / 7$. Of course these $\alpha, \beta$ will have the property that $1, \alpha$, $\beta$ is an integral basis for $Q(\theta)$.

A listing of the first 1000 partial quotients for $\theta$ is given in [13, p. 128]. We find that $a_{57}=60, a_{58}=a_{59}=1, a_{60}=50$. If we apply the method of [9] to this pattern, we produce numbers $\alpha, \beta$ such that $c_{1}(\alpha, \beta)>.285$. This numerical result was needed in $\S 3$. Thus $L^{\prime}>.285$. By more extensive calculations we could achieve lower bounds still closer to $2 / 7=.2857 \ldots$

7. Concluding remarks. For each real number $\alpha$, let $c(\alpha)$ denote the one-dimensional Diophantine approximation constant defined in the Introduction. The set of all $c(\alpha)$ is usually called the Lagrange spectrum (and a closely related set is called the Markoff spectrum). Much is known about the distribution of the numbers $c(\alpha)$. As was remarked in the Introduction, the largest possible value of $c(\alpha)$ is $1 / \sqrt{5}$. It is known that this is an isolated value, and that there is an infinite sequence of other isolated values between $1 / \sqrt{5}$ and the largest limit point $1 / 3$. 
We have very little knowledge about the distribution of the numbers $c_{1}(\alpha, \beta)$ and $c_{2}(\alpha, \beta)$. Indeed, it is only conjecture (see the Introduction) that $2 / 7$ is the least upper bound for these numbers. If we confine ourselves to numbers $\alpha, \beta$ such that $1, \alpha, \beta$ is a basis for a real cubic field, then $2 / 7$ is the least upper bound, but it is not attained (see $\S 4$ ). This is quite different from the situation, described above, for the one-dimensional constants $c(\alpha)$.

It is very likely that no result like Theorem 2 is true if the restriction to integral bases is removed. In particular, it is very likely that for arbitrary bases $1, \alpha, \beta$ of real cubic fields, $c_{1}(\alpha, \beta)$ does not tend to 0 as the field discriminant tends to infinity. The reason for believing this is that the corresponding result in the one-dimensional case is false (Lemma 5 below), and there is no reason to suppose that the two-dimensional case is any simpler.

LEMMA 5. There exists a sequence $\alpha_{1}, \alpha_{2}, \ldots$ of real quadratic irrationals such that the $\alpha_{n}$ belong to infinitely many different real quadratic fields and such that $c\left(\alpha_{n}\right) \leq \sqrt{12}$ for $n=1,2, \ldots$

Proof. Let $f_{1}=f_{2}=1, \ldots, f_{n}=f_{n-1}+f_{n-2}(n \geq 3)$ denote the sequence of Fibonacci numbers. A calculation shows that

$$
\left(f_{n+2} / f_{n}\right)^{1 / 2}=\left[1, \overline{1_{n-1}, 2}\right] \quad(n=1,2, \ldots),
$$

where the bar indicates the period of the continued fraction on the right and $1_{n-1}$ stands for $n-1$ repetitions of the digit 1 . We define $\alpha_{n}=$ $\left(f_{n+2} / f_{n}\right)^{1 / 2}(n=1,2, \ldots)$; then it is easily seen that $c\left(\alpha_{n}\right) \leq \sqrt{12}$ for all $n$. It follows from well known results (e.g., the Lucas law of repetition of primes in the Fibonacci sequence - see for example [14, pp. 421-422]) that the largest prime factor in $f_{n}$ tends to $\infty$ as $n \rightarrow \infty$; this shows that the numbers $\alpha_{n}$ belong to infinitely many different quadratic fields.

Of course the $\alpha_{n}$ in Lemma 5 are not all algebraic integers. Indeed, the analogue of Theorem 2 is true for the numbers $c(\alpha)$, since it is easily proved that if $\alpha$ is an algebraic integer of the real quadratic field $Q(\sqrt{d})$, then $c(\alpha) \leq 1 / d^{1 / 2}$.

\section{REFERENCES}

1. W. W. Adams, Simultaneous Diophantine approximations and cubic irrationals, Pacific J. Math., 30 (1969), 1-14.

2 . The best two-dimensional Diophantine approximation constant for cubic irrationals, Pacific J. Math., 91 (1980), 29-30.

3. I. O. Angell, A table of totally real cubic fields, Math. Comput., 30 (1976), 184-187. 
4. J. W. S. Cassels, Simultaneous Diophantine approximation, J. London Math. Soc., 30 (1955), 119-121.

5. __ An Introduction to the Geometry of Numbers, Springer-Verlag, Berlin, 1959.

6. J. H. H. Chalk, On the positive values of linear forms, Quart. J. Math., (Oxford) (2) 18 (1947), 215-227.

7. T. W. Cusick, Formulas for some Diophantine approximation constants, Math. Annalen, 197 (1972), 182-188.

$8 . \quad$, Formulas for some Diophantine approximation constants II, Acta Arith., 26 (1974-75), 117-128.

9. $\quad$ The two-dimensional Diophantine approximation constant, Monats. Math., 78 (1974), 297-304.

10. H. Davenport, Simultaneous Diophantine approximation, Proc. London Math. Soc., (3) 2 (1952), 406-416.

11. On a theorem of Furtwängler, J. London Math. Soc., 30 (1955), 186-195.

12. H. Davenport and K. Mahler, On simultaneous Diophantine approximation, Duke Math. J., 13 (1946), 105-111.

13. S. Lang and $\mathrm{H}$. Trotter, Continued fractions for some algebraic numbers, J. Reine Angew. Math., 255 (1972), 112-134.

14. D. H. Lehmer, An extended theory of Lucas' functions, Ann. Math., (2) 31 (1930), 419-448.

15. A. C. Woods, The asymmetric product of three homogeneous linear forms, Pacific J. Math., 93 (1981), 237-250.

Received July 30, 1981.

SUNY AT BUFFALO

BUfFalo, NY 14214 



\section{PACIFIC JOURNAL OF MATHEMATICS}

EDITORS

Donald BABBITT (Managing Editor)

University of California

Los Angeles, CA 90024

Hugo Rossi

University of Utah

Salt Lake City, UT 84112

C. C. Moore and Arthur Ogus

University of California

Berkeley, CA 94720
J. DugunduI

Department of Mathematics

University of Southern California

Los Angeles, CA 90089-1113

R. FinN and H. SAmelson

Stanford University

Stanford, CA 94305

\section{ASSOCIATE EDITORS}
R. ARENS
E. F. BECKENBACH
B. H. NEUMANN
F. WolF
K. YoshidA (1906-1982)

\section{SUPPORTING INSTITUTIONS}

UNIVERSITY OF ARIZONA

UNIVERSITY OF BRITISH COLUMBIA

CALIFORNIA INSTITUTE OF TECHNOLOGY

UNIVERSITY OF CALIFORNIA

MONTANA STATE UNIVERSITY

UNIVERSITY OF NEVADA, RENO

NEW MEXICO STATE UNIVERSITY

OREGON STATE UNIVERSITY
UNIVERSITY OF OREGON

UNIVERSITY OF SOUTHERN CALIFORNIA

STANFORD UNIVERSITY

UNIVERSITY OF HAWAII

UNIVERSITY OF TOKYO

UNIVERSITY OF UTAH

WASHINGTON STATE UNIVERSITY

UNIVERSITY OF WASHINGTON 


\section{Pacific Journal of Mathematics}

Vol. 105, No. 1 September, 1983

Kenneth F. Andersen, On the transformation of Fourier coefficients of

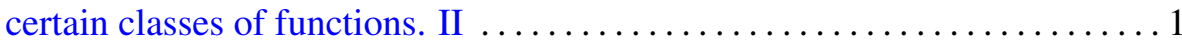

Gavin Brown, Irving Leonard Glicksberg and Edwin Hewitt, Indicator functions with large Fourier transforms $\ldots \ldots \ldots \ldots \ldots \ldots \ldots \ldots \ldots \ldots$

Shih-Sen Chang, Some random fixed point theorems for continuous random

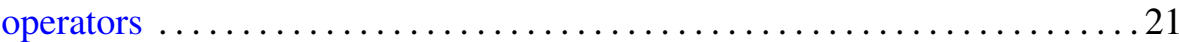

K. C. Chattopadhyay and Olav Njstad, Quasiregular nearness spaces and

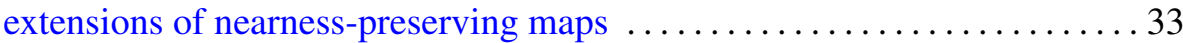

Thomas W. Cusick, The two-dimensional Diophantine approximation

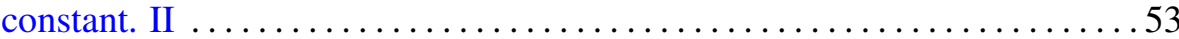

Eric Karel van Douwen and Jan van Mill, Spaces without remote points . . .669 Hector O. Fattorini, Convergence and approximation theorems for

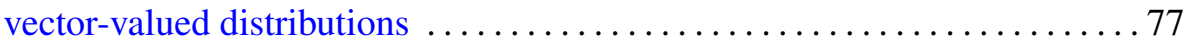

John J. F. Fournier and Louis Pigno, Analytic and arithmetic properties of

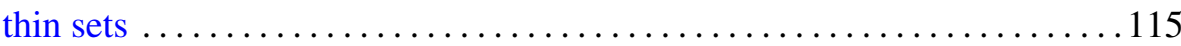

David Goss, On a new type of $L$-function for algebraic curves over finite

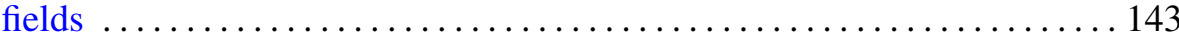

Douglas Austin Hensley, Lattice vertex polytopes with interior lattice

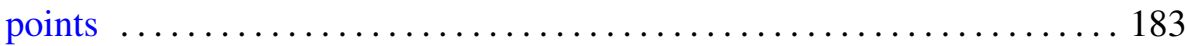

J. K. Kohli, Monotone extensions of mappings and their applications ...... 193

John C. Morgan, II, On equivalent category bases . . . . . . . . . . . . 207

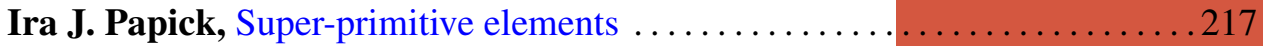

José Luis Rubio de Francia and José Luis Torrea, Vector extensions of

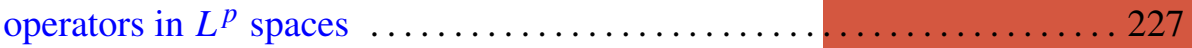

Mark Phillip Thomas, Closed ideals of $l^{1}\left(\omega_{n}\right)$ when $\left\{\omega_{n}\right\}$ is star-shaped $\ldots 237$ 